\title{
Government Bus Tracking With Passenger Details
}

\author{
Rathnavel. K, Selvasundar. K, Jamuna. V
}

\begin{abstract}
This paper proposes an alternative to the conventional public transport tracking systems which uses GPS. The proposed model uses LoRa wireless transmission to communicate between the bus stops and a base station. The buses are equipped with $R F$ transmitters, which send out data regarding the bus identity, continuously. $R F$ receivers placed in the bus stop, detects this bus when it is in range, and relays this information to the base station instantly through LoRa communication. The LoRa receiver in the base station collects the transit information from all such bus stops in its range, modifies it as per requirement, and stores all necessary information in a database. The prototype built, only cost one-seventh of the cost required to implement a conventional tracking system, and consumed much less power as well. Such a system has minimal dependence on the number of buses being used. Hence, the system can be scaled at minimal costs
\end{abstract}

\section{Index Terms: Public Transport, LoRa, GPS, RF, Nano.}

\section{INTRODUCTION}

Transport system in India consists of transport by land, water, and air. Public transport remains the primary mode of transport for most Indian citizens, and India's public transport systems are among the most heavily used in the world. Motor vehicle population in India is low as per international standards, with only 24.85 million cars on the nation's roads as per 2013 records. In total, about 21 percent of households have two wheelers whereas only 4.7 percent of households in India have cars / jeeps / vans as per the 2011 Census. Despite this, the number of deaths caused by traffic is amongst the highest in the world and increasing. The automobile industry in India is currently rapidly growing with an annual production of over 4.6 million vehicles, with an annual growth rate of $10.5 \%$ and vehicle volume is expected to rise greatly in the future.

\section{A. Analysis}

The development of city bus systems can be significantly strengthened by lessons learnt through the implementation of innovative practices and challenges faced in urban India. We start by evaluating the relative successes and challenges of city bus reforms initiated by some of EMBARQ India's partners - Brihan Mumbai Electricity Supply and Transport (BEST) Undertaking, Bangalore Metropolitan Transport

Revised Manuscript Received on July 22, 2019.

Rathnavel.K, Electronics and Instrumentation Engineering, Jerusalem College of Engineering, Chennai, India.

Selvasundar.K, Electronics and Instrumentation Engineering, Jerusalem College of Engineering, Chennai, India.

Jamuna.V, Electronics and Instrumentation Engineering, Jerusalem College of Engineering, Chennai, India.
Corporation (BMTC), Atal Indore City Transport Services Ltd. (AICTSL), Bhopal City Links Limited (BCLL) and the Metropolitan Transport Corporation, Chennai (MTC). While EMBARQ India was actively engaged in some of these initiatives, other observations are based on our collaboration with partners.

Pilot initiatives and bus reforms in recent years reinforce that city bus systems will continue to be the backbone of urban mobility in India. It is therefore imperative that cities across India focus on sustainable transport and set targets to achieve a higher modal share of public transport. Buses take up over $90 \%$ of public transport in Indian cities (Pucher, Korattyswaroopam and Ittyerah 2004), and serve as an economical and convenient mode of transport for all classes of society. There are approximately 35,000 buses operational in urban areas. Of this, eight of the bigger cities - Delhi, Kolkata, Mumbai, Chennai, Bangalore, Hyderabad, Ahmadabad, and Pune - account for 80 percent of all buses.

Currently, the bus Modal share of these cities varies from one percent (Surat) to 43 percent (Bangalore), as seen in Figure 4 . The challenge is to retain the bus modal share and further increase the modal share of public transport through various initiatives. EMBARQ India recommends that by 2020, bus transport must comprise of at least half of the mode share of all motorized trips in Tier 1 cities and at least one-third of the mode share in Tier 2 and 3 cities.

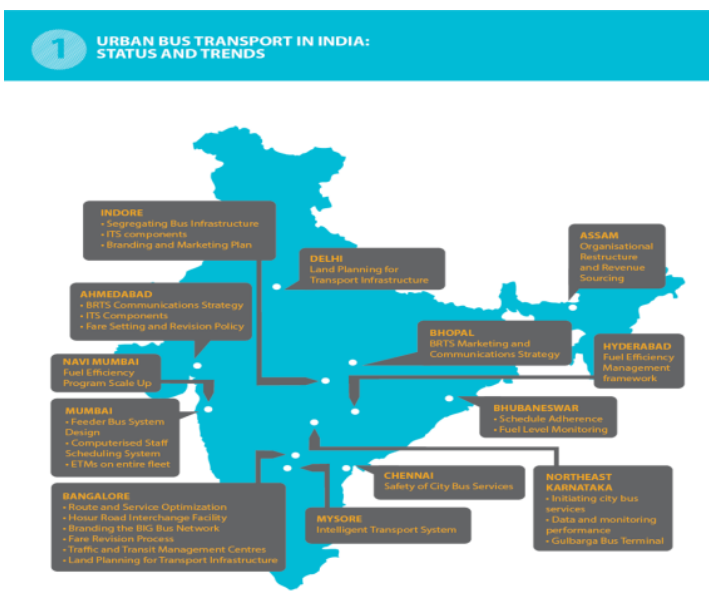

Fig 1 Bus Transport Mode Shares (excluding non-motorized trips) in Indian cities

Following the launch of the Version 1 Guidebook,

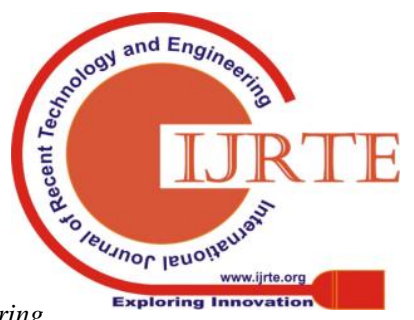


EMBARQ India continued to work closely with numerous transport undertakings and participate in several of the major reforms across the bus industry. The following section highlights some of these phenomenal and unprecedented changes.

\section{PROPOSED SYSTEM}

\section{A. Communication Protocol}

The proposed system comprises of Lora, Microcontrollers and IoT modules and Display modules.
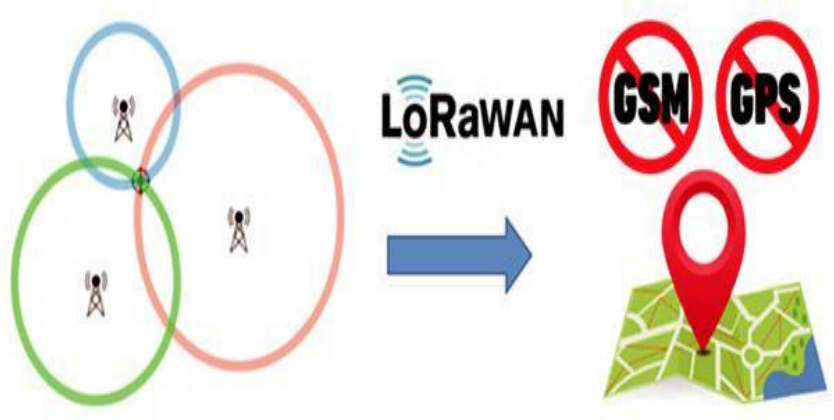

Fig 2 LoRaWan

LoRaWAN presents interesting features, so it becomes a powerful technology in geolocation applications. For instance, the long range that can be reached, up to $15 \mathrm{~km}$ in rural areas and $5 \mathrm{~km}$ in urban areas due to the good sensitivity of the receivers $(-130 \mathrm{dBm})$. Consequently, the total path of the link budget could reach up to $155 \mathrm{~dB}$ [3]. Another interesting characteristic is the bandwidth, which is bigger than other IoT technologies in LPWANs, so it is better to distinguish different paths from the same signal (useful for tracking capabilities in urban scenarios where reflections are present) [4]. Furthermore, various studies about LoRa technology for geolocation have been carried out in recent years, in which Sagemcom obtained good accuracy results (up to 4 meters). However, 42 gateways were used in a hexagonal layout to improve the results and the signal from the end-node was received by at least 10 gateways, when the data rate was at the highest [5]. Finally, LoRaWAN is an open source technology, there is information about its implementation, layers, packet structure, protocols of communications and other relevant features.

\section{B. Block Diagram}

LoRa (short for long range) is a spread spectrum modulation technique derived from chirp spread spectrum (CSS) technology. Semtech's LoRa devices and wireless radio frequency technology (LoRa Technology) is a long range, low power wireless platform that has become the de facto technology for Internet of Things (IoT) networks worldwide. LoRa Technology enables smart IoT applications that solve some of the biggest challenges facing our planet: energy management, natural resource reduction, pollution control, infrastructure efficiency, disaster prevention, and more. Semtech's LoRa Technology has amassed over 600 known uses cases for smart cities, smart homes and buildings, smart agriculture, smart metering, smart supply chain and logistics, and more.
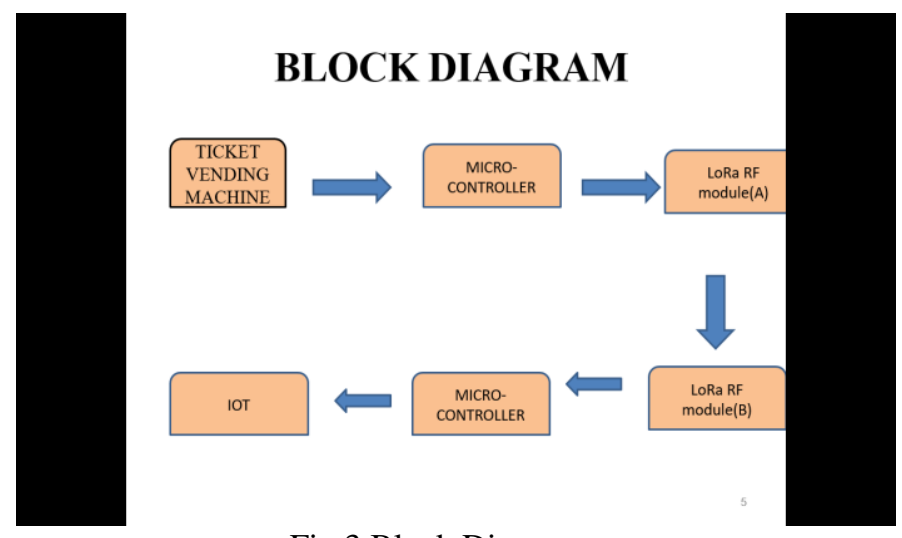

Fig 3 Block Diagram

\section{HARDWARE SECTION}

This section comprises of processor used for this project which is discussed as follows

\section{A.NodeMCU ESP8266 Controller}

NodeMCU is an open source IoT platform. It includes firmware which runs on the ESP8266 Wi-Fi SoC from Espress if Systems, and hardware which is based on the ESP-12 module. The term "NodeMCU" by default refers to the firmware rather than the development kits.

\section{B.Arduino Nano}

Arduino is an open-source electronics prototyping platform based on flexible, easy-to-use hardware and software. It's intended for artists, designers, hobbyists, and anyone interested in creating interactive objects or environments. Arduino can sense the environment by receiving input from a variety of sensors and can affect its surroundings by controlling lights, motors, and other actuators. The microcontroller on the board is programmed using the Arduino programming language (based on Wiring) and the Arduino development environment (based on Processing).

\section{SYSTEM DESIGN}

The overall system consisted mainly of four blocks: an end-node, four gateways, The Things Network (TTN) and a third-party application.

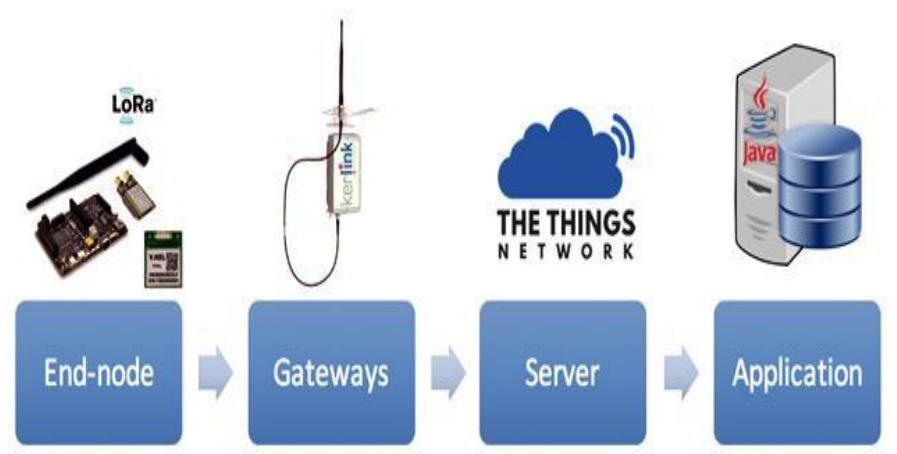

Fig 4 Elements of the whole system

The end-node sent the data over the air using the

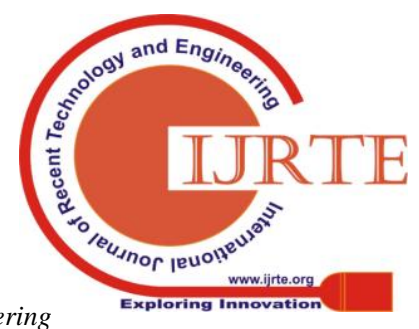


LoRaWAN protocol and the gateways that were close enough (around $5 \mathrm{~km}$ ) received the data. Then, the gateways forwarded the packets via UDP/IP to TTN, together with information from the received signal such as the exact time when the packet was received, the RSSI, the working frequency, etc. Afterwards, TTN processed the data from the different gateways and routed the messages to the application using a Message Queue Telemetry Transport (MQTT) client. Finally, the algorithm to estimate the position was applied in a third- party application.

\section{A. End-node}

The end-node was responsible for sending the data acquired from a GPS receiver over the air through a LoRaWAN module. A GPS module was used to be able to benchmark and reference the performance of the investigated geo location method. A Wasp mote board was the main device of the end-node, so it processed and gathered information from the other modules. The Wasp mote's core is based on the Atmel ATmega1281 microcontroller and is suitable for IoT applications. The modular architecture allows to integrate only the modules needed in each situation. Wasp mote was selected as the core processor because of its low power consumption, which was one of the main requirements of the system. The feasibility for allowing to connect a LoRaWAN module was also relevant for choosing this device. The GPS coordinates were transmitted approximately every 10 seconds over the LoRaWAN and the Wasp mote parsed the coordinates and transmitted them as a payload in the packet.

\section{B. Gateways}

The main function of the four gateways was to route the data received from the end-node to the server via UDP / IP. In order to estimate the location of the device, the received time of the packet from each gateway was needed to apply the multilateration algorithm. There are several gateways supporting LoRa technology on the market. The chosen one was Kerlink because a GPS receiver is embedded in the gateway. Therefore, all gateways are synchronized by using the timestamp from the GPS satellites.

\section{The Things Network}

TTN [10] was responsible for decoding data from the four gateways and transmitting it to the third-party application. It was important that the four packets from the different gateways, with the same payload but with different times, arrived at the network server. The most relevant feature for the algorithm computation was the value of the time field in the JSON which was sent. TTN is an open-source platform and allows a wide variety of third-parties to be connected. TTN offers a feature full and useful web interface to see the data that has been sent. However, if the goal is to process and analyze such information, a communication with a third-party application is required. Thus, a MQTT client was used to forward the data to a Java application.

\section{Third-party application}

The third-party application consisted mainly of two parts: a Java application and a MySQL database. The main function was to obtain the data from TTN, parse it and insert it in the database for processing in the following step. As already stated, MQTT client was used to establish the communication with the server. The MQTT is a machine-to-machine (M2M) connectivity protocol which is designed as an extremely lightweight publish and subscribe messaging transport. TTN uses MQTT to publish device activations and messages, but also allows the user to publish a message for a specific device in response.

\section{Tests AND RESUlts}

The gateways were distributed around the area taking into account some aspects. First of all, they had to form a four-sided polygon, each side with a length of around 2 to 3 $\mathrm{km}$. The location was also selected according to the altitude, since the higher the antenna, the larger the coverage. This improves the sensitivity of the device, because if the height of the antenna is doubled, a gain of $6 \mathrm{~dB}$ is achieved considering the flat Earth model.

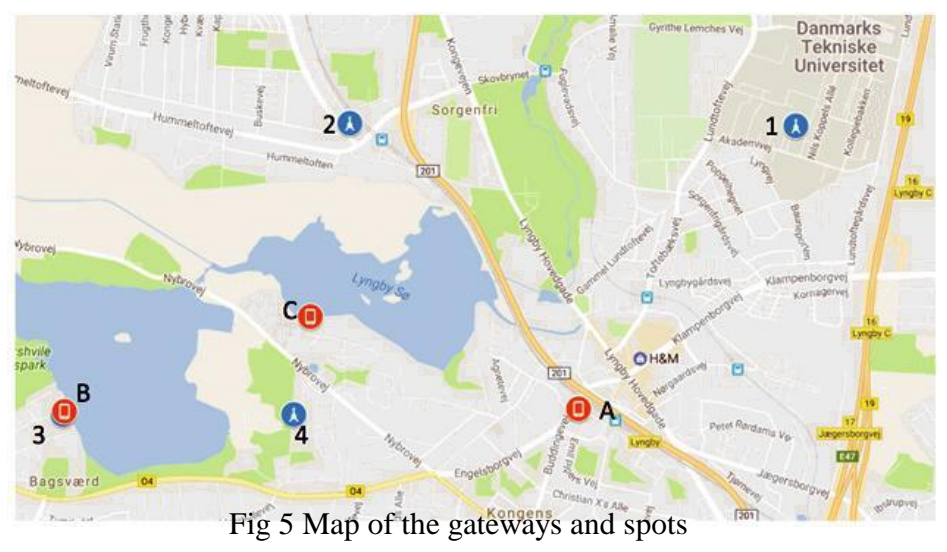

\section{A. Open Space}

Here the set up equipment is on a location which has at least one kilometer with a free line of sight. Then the end-node device is moved further and further away from the gateway and on each 300 meters measurement is done. As soon as a single data-package is lost in the transmissions the step is decreased to 100 meters. The process is continued until either 0 packages are received or we run out of space that still maintain free line of sight. In Fig 6 the location and distances can be seen. The gateway is stationary while the end-node device gets positioned at different distance.

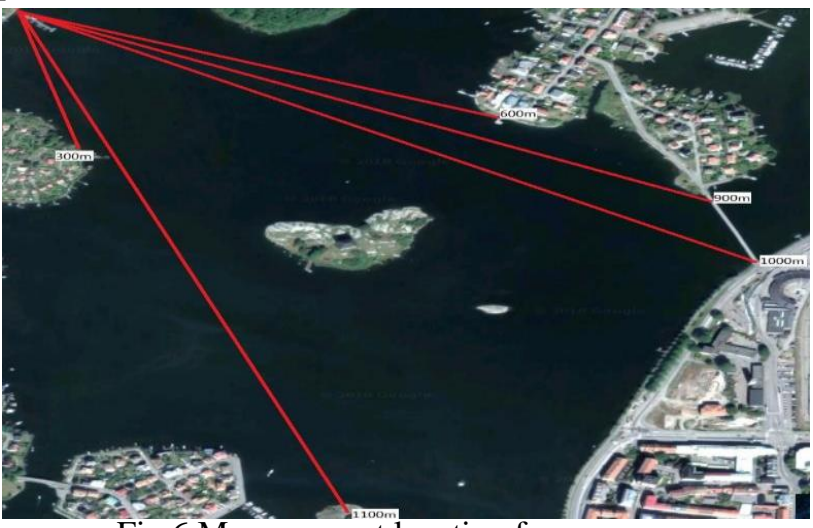

Fig 6 Measurement location for open space

\section{B. Dense Forest}

On this part a location where the terrain was relatively flat and with dense

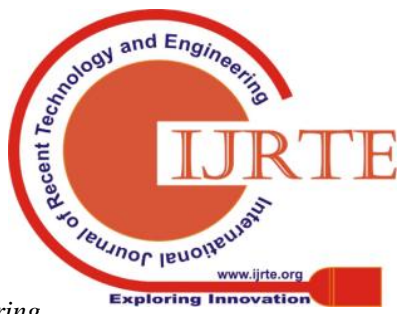




\section{Government Bus Tracking With Passenger Details}

vegetation is found. Then the measurements starting at 300 meters are done. From then the distance is increased by 100 meters each step then expect the signal to drop more drastically compared to having free line of sight. Fig 7 shows the location and setup of gateway and end-node device. Gateway is stationary in the bottom right while the device gets moved further away on a straight line.

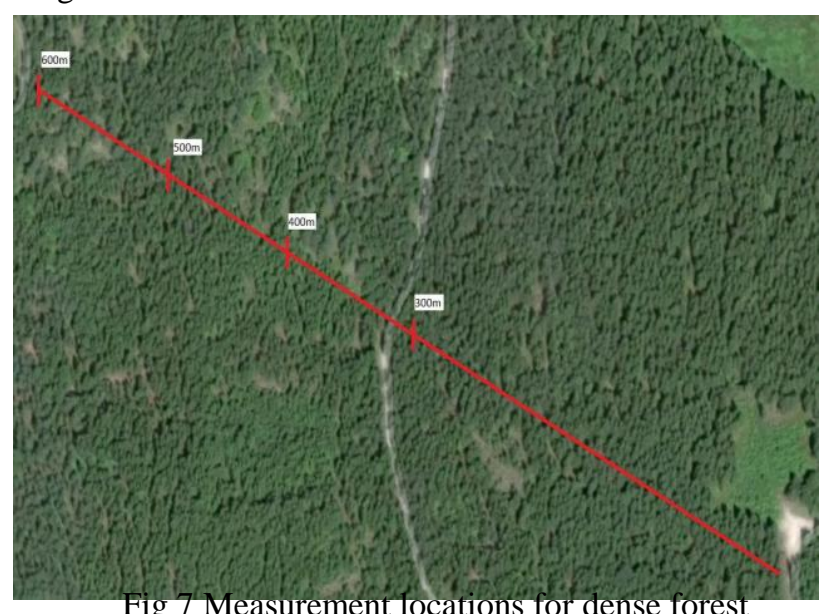

VI CONCLUSION

This whole paper mainly focuses on two things. The First thing is the concept of developing a simple system for bus seat availability in public transportation. Second section includes the development of application for the ease usage of people. Due to higher population and its increasing factors, Buses are the most likely mode of transportation, decreasing the comfort of journey. Our system enables easy and conform tracking and travelling of public transport system. Though there are many tracking system for MTC buses available our system enables user friendly function since it is developed in the vision of ease maintenance.

\section{REFERENCES}

[1] A. Augustin, J. Yi, T. Clausen, and W. Townsley, "A Study of LoRa: Long Range \& Low Power Networks for the Internet of Things," Sensors, vol. 16, no. 9, p. 1466, 2016.

[2] A. Note, "Gateway to Server Interface LoRaWAN Network Server Demonstration : Gateway to Server Interface Definition Gateway to Server Interface," no. March, pp. 1-17, 2015.

[3] L. Mainetti, L. Patrono, A. Secco, and I. Sergi, "An IoT-aware AAL system for elderly people," 2016 Int. Multidiscip. Conf. Comput. Energy Sci. Split. 2016, 2016.

[4] Bankov, D.; Khorov, E.; Lyakhov, A. (November 2016). "On the Limits of LoRaWAN Channel Access". 2016 International Conference on Engineering and Telecommunication (EnT): 10-14. doi:10.1109/ent.2016.011

[5] Matthew Knight; Balint Seeber (2016). "Decoding LoRa: Realizing a Modern LPWAN with SDR"

[6] "Semtech Selected for Smart Irrigation System | San Fernando Valley Business Journal". sfvbj.com.

[7] Mu-Hyun, Cho. "SK Telecom launches LoRa-based fire detection solution". ZDNet.

[8] K.Rathnavel "Accident Alert and Intercommunication System using Arduino.” In IJARMATE, Vol 3, Special Issue 13, March 2017.

[9] K.Rathnavel "Blind User Wearable Audio Assistance for Outdoor Navigation using PI based Image Processing” in IJSRR Journal, Vol 7, Issue 4, April 2018.

[10] K.Selvasundar, "Industrial Intelligent line follower vehicle with colour track detection", International journal of advanced research in management architecture, Technology and Engineering .Volume 3, Special issue 13, March 2017.

[11] K.Selvasundar, "Realization of Control Strategy for a Non-linear System using FGPA", International Journal of Scientific Research and Review, Volume 7, Issue 4, 2018 